\title{
Precision of thermonuclear supernovae as distance indicators
}

\section{Inma Domínguez*}

Universidad de Granada, Spain E-mail: inma@ugr.es

\section{Eduardo Bravo}

Universitat Politecnica de Catalunya, Barcelona, Spain

E-mail: eduardo.bravo@upc.es

\section{Luciano Piersanti}

INAF-Osservatorio Astronomico di Collurania, Teramo, Italy

E-mail: piersanti@oa-teramo.inaf.it

\section{Oscar Straniero}

INAF-Osservatorio Astronomico di Collurania, Teramo, Italy

E-mail: stranierodoa-teramo.inaf.it

\section{Peter Höflich}

Florida State University, Tallahassee, USA

E-mail: paheastro.physics.fsu.edu

\section{Jordi Isern}

Institut de Ciencies de l'Espai, CSIC, Barcelona, Spain

E-mail: isern@ieec.uab.es

\section{Amedeo Tornambé}

INAF-Osservatorio Astronomico di Roma, Italy

E-mail: tornambedoa-teramo.inaf.it

Supernovae: lights in the darkness (XXIII Trobades Científiques de la Mediterrània) October 3-5 2007

Mao, Menorca, Spain

\footnotetext{
* Speaker.
} 


\section{Abstract}

Observations of thermonuclear supernovae (SNe Ia) at high-redhift (z) show that the Universe is dominated by the Dark Energy term, characterized by its negative pressure. SNIa Hubble diagram, including nowadays 200 of these objects up to $\mathrm{z}=1.8$, strongly favours a decelerated expansion of the Universe up to $\mathrm{z}=0.5$ and an accelerated one at current time. Taken all current data together (SNe Ia, Cosmic Microwave Background, Barionic Acoustic Oscillations and Large Scale Structure) the fraction of the dark energy to the critical density is $70 \%$ and its EOS, $w=\mathrm{P} / \rho$, is compatible with the cosmological constant or vacuum energy, $w=-1$, without a temporal dependence. However, any further attempt to characterize this EOS based on SNe Ia requires to reduce the present scatter in the Hubble diagram by a factor of 10. Ultimately, systematic effects, like extinction and evolution with $\mathrm{z}$, and uncontrolled intrinsic dispersion of the light curve shapes will limit the precision by which SNe Ia could be used to probe the structure of the Universe and its time evolution. Moreover, observations indicate that brighter SNe Ia occur only in systems with ongoing star formation and that the majority of SNe Ia comes from young progenitors. In this work we focus on the scatter of the maximum luminosity of SNe Ia as determined by possible variation of some properties of their progenitors, namely: (a) mass and metallicity of the progenitor of the exploding white dwarf and (b) rotational velocity in the framework of double degenerate systems. We obtain differences up to 0.2 magnitudes at maximum, which are significant to explore the nature of the dark energy.

\section{Introduction}

A decade ago the observations of SNe Ia at $\mathrm{z} \sim 0.5$ showed the unexpected result of the acceleration of the expansion of the Universe (Schmidt, 1998; Perlmutter, 1999). Nowadays the SNIa Hubble diagram, including $200 \mathrm{SNe}$ up to $\mathrm{z}=1.8$, strongly favours a decelerated expansion of the Universe up to $\mathrm{z}=0.5$ and an accelerated one at current time (Riess et al. 2007). All the available data (SNe Ia, Cosmic Microwave Background, Baryon Acoustic Oscillations and Large Scale Structure) are compatible with a $70 \%$ of dark energy with an EOS, $w=P / \rho$, in which $w=-1$ (cosmological constant or vacuum energy) without a temporal dependence.

At the beginning, SNe Ia were considered to be very similar, if not identical, each other, but it became soon evident that this hypothesis was not correct since differences up to 2 magnitudes (in V) were observed at the maximum epoch. However, in 1993 Phillips showed that for a number of near and well observed SNe Ia, whose distance was a-priori determined by means of bona fide distance indicators, a linear correlation exits between maximum luminosity and decline rate of luminosity after maximum. Basing on this evidence, these Supernovae can be considered once again as an homogeneous class and they can be used as standardized candles (Phillips 1993, Hamuy et al. 1996, Phillips et al. 1999). However, key questions remain still open: (a) Is the correlation universal, so that it can be safely applied to SNe Ia on cosmological distances ? and (b) if the answer to the previous question is positive, the present scatter in the Hubble diagram has to be reduced by a factor of 10 to characterize the Dark Energy.

It is clear that systematic effects, like extinction and evolution with $\mathrm{z}$, and uncontrolled intrinsic dispersion of the light curve shapes will limit the precision by which SNe Ia could be used as 
distance indicators..

Recent observations, at low and high z, confirm that brighter SNe Ia occur only in systems with ongoing star formation and that the majority of SNe Ia comes from young stellar populations. These results imply that SNe Ia arise from progenitors with evolutionary times that differ in 2$3 \mathrm{Gyr}$, and also that the differences in the light curves are expected to depend on the progenitor properties (Branch et al. 1996; Hamuy et al., 1995, 1996, 2000; Ivanov et al. 2000; Sullivan et al. 2006, Mannucci et al. 2005, 2006).

In this work we focus on the scatter of the maximum luminosity of SNe Ia as determined by possible variation of some properties of their progenitors, namely: (a) mass and metallicity of the progenitor of the exploding Chandrasekhar-mass WD and (b) rotational velocity in the framework of the merging of two WDs. This study is not straightforward as we are still far from a complete understanding of Type Ia supernovae, in particular with respect to the identification of the progenitors and the explosion mechanism. We will focus on the progenitors, although the explosion mechanism and its relation with the progenitor could well be fundamental.

\section{The explosion mechanism}

Many studies have shown that the delayed detonation explosion (Khokhlov 1991) of a Chandrasekhar mass $\left(\mathrm{M}_{C h}\right)$ WD satisfactorily explains most of the observed properties: the evolution of the LCs and spectra in the optical and in the IR, and also, the correlations observed, including the crucial maximum decline relation (Fisher et al. 1995; Höflich 1995; Höflich \& Khokhlov 1996; Wheeler et al. 1998; Lentz et al. 2000; Höflich et al. 2002, 2003). For these reasons, we have adopted this explosion mechanism and we have kept constant the transition density -the density at which the change from a deflagration to a detonation takes place-, and compute the explosions and light curves (for details see Domínguez et al. 2001, Bravo et al. 2003, 2006; Badenes et al. 2003).

\section{Mass and metallicity of the progenitor of the exploding WD}

We have considered the entire range of potential progenitors, with 1.5-7 $\mathrm{M}_{\odot}$ and metallicities between $\mathrm{Z}=0$ and 0.02 . The pre-supernova evolution (starting from the pre-main sequence phase) is simulated by means of a 1D hydrostatic code, the FRANEC code (Chieffi et al. 1998; Domínguez et al. 1999; Straniero et al. 1997; Chieffi et al. 2001). Starting from the computed WD structures, detailed model calculations have been performed for the hydrodynamic explosion, nucleosynthesis and LCs (Domínguez el al. 2001 and references therein). The single degenerate scenario for the progenitors has been assumed (accretion of H-rich matter onto the WD).

We could distinguish two regions in the exploding structure: the inner one, the original WD (with masses between 0.55 and $0.99 \mathrm{M}_{\odot}$, corresponding to initial masses between 1.5 and $7 \mathrm{M}_{\odot}$ ) and the external one, formed by the accreted matter. The $\mathrm{C} / \mathrm{O}$ rate is determined by the previous He-burning phases. Initially carbon is produced via the $3 \alpha$ reactions and once sufficient ${ }^{12} \mathrm{C}$ is synthesized, the ${ }^{12} \mathrm{C}(\alpha, \gamma){ }^{16} \mathrm{O}$ reaction becomes competitive with the $3 \alpha$, and carbon is partially burned into oxygen. The amount of $\mathrm{C} / \mathrm{O}$ is greater $(\sim 1)$ in the accreted matter because in these layers the ${ }^{12} \mathrm{C}(\alpha, \gamma){ }^{16} \mathrm{O}$ reaction does not have enough time to destroy ${ }^{12} \mathrm{C}$ (unlike the situation 
during central convective He-burning). This higher $\mathrm{C} / \mathrm{O}$ rate in the previously accreted layers is critical for the final average $\mathrm{C} / \mathrm{O}$ within the exploding structure.

More massive progenitors, accrete less mass and finally the average $\mathrm{C} / \mathrm{O}$ is smaller. As a consequence, the ${ }^{56} \mathrm{Ni}$ mass synthesized during the explosion is smaller and the $\mathrm{SN}$ is dimmer, showing smaller expansion velocities of the ejecta (up to $2000 \mathrm{~km} / \mathrm{s}$ considering the whole range). Notice that the correlation between LC shape and expansion velocity could be used to further reduce the scatter in the calibration relation. The size of the obtained effect, $\Delta \mathrm{M}_{\max } \sim 0.2 \mathrm{mag}$, is critical for the reconstruction of the cosmological equation of state.

At the other hand, the chemical composition of the CO WD is not sensitive to the initial $\mathrm{Z}$ of its progenitor (for details see Domínguez el al. 2001) because the mass of the CO core midly depends on the initial metallicity. For this reason, the ${ }^{56} \mathrm{Ni}$ mass and the maximum luminosity do not depend on the metallicity. However, the metallicity alters the isotopic composition of the outer layers of the ejecta and less Fe-lines contribute to the opacity. This effect is important at short wavelengths, changing the intrinsic colour index B-V by up to -0.06 mag; moreover, it alters the fluxes in the U band and the UV (Höflich et al. 1998). The change in B-V is critical if extinction corrections are applied. The offset in the maximum-decline calibration is not monotonic in $\mathrm{Z}$ and, in general, remains smaller than 0.07 mag.

\section{Rotational effects in Double Degenerate Systems}

Although in the previous sections we have assumed that the WD achieves the $\mathrm{M}_{C h}$ by accretion from a companion, this is still a matter of hot debate. Accretion of $\mathrm{H}$ or $\mathrm{He}$ from a non-degenerate companion at low accretion rates gives rise to a nova, while at high accretion rates it leads to the expansion of the structure and mass loss; thus, the range of accretion rates suitable to produce the growth in mass of the WD is very narrow.

We consider the merging of two CO WDs (Double Degenerate scenario or DD) in which the smaller WD completely disrupts forming a CO thick disk around the more massive companion. For these systems the accretion rate is so high that off-center ignition of C-burning occurs well before the accreting WD could attain the $\mathrm{M}_{C h}$ (Saio \& Nomoto 1985).

This situation changes if the effects of rotation are taken into account in the evolution of the accreting WD (Piersanti et al. 2003a and 2003b, although see Saio \& Nomoto, 2004). We have assumed that the two WD rotate at the orbital frequency and we have assumed instantaneous redistribution of angular momentum in the accreting WD (rigid rotation). In this situation the accretion rate decreases and the off-center ignition of carbon burning is prevented. Then, a balance is reached between angular momentum lost via gravitational wave radiation and angular momentum gained from the accreted matter. Due to the lifting effect of rotation, this balance allows the WD to increase its mass over the canonical $\mathrm{M}_{C h}$ and up to the limit for rigidly rotating degenerate structures $\left(1.5 \mathrm{M}_{\odot}\right)$. In this way it is possible to obtain exploding object with total mass in the range 1.4-1.5 $\mathrm{M}_{\odot}$, depending on the total mass of the initial DD system.

Models of masses ranging between 1.4 and $1.5 \mathrm{M}_{\odot}$ have been analyzed. Differentially rotating WDs may be stable up to several solar masses but here rigid body rotation has been assumed so that the corresponding Chandrasekhar limit is $\sim 1.5 \mathrm{M}_{\odot}$. 
As described in detail by Domínguez et al. (2006) the properties of models with different total mass are very similar each other. In fact, the luminosity depends on the amount of ${ }^{56} \mathrm{Ni}$ synthesized during the explosion, which, on its side, depends mainly on the density at which the burning occurs. The latter is determined by the expansion during the deflagration phase whose time length is determined by the transition density which is maintained constant in our experiments.

Nonetheless, rotation and braking efficiency in the pre-ignition stage produce a spread in binding energies and ignition densities at the explosion time. According to the numerical results, more massive models have greater binding energies but also higher ignition densities. Hence, a larger amount of energy has to be spent to unbind the WD, thus implying a smaller final kinetic energy and a larger completely incinerated zone, producing more ${ }^{56} \mathrm{Ni}$. However, electron captures are also favored at higher densities and less ${ }^{56} \mathrm{Ni}$ would be produced in the inner regions. In summary, the production of ${ }^{56} \mathrm{Ni}$ does not show a monotonic dependence with the ignition density, binding energy and total mass.

\section{Conclusions}

This study of the influence of the progenitor, considering the mass and composition of the WD progenitor and taking into account the rotation of the accreting WD, reveals differences in the maximum magnitudes, for similar decline rates, of up to $0.2 \mathrm{mag}$; that is, differences of the same order of the present dispersion in the maximum-decline relation. Therefore, current estimates on the cosmological evolution of the Universe remain valid also in the light of these new possible interpretation of type Ia SNe progenitors. The same occurs for the chemical evolution, as the yields are only marginally changed.

However, taking into account that an accuracy of about 0.02 mag is required to characterize the nature of the dark energy, it is clear that the next step is to reduce the present scatter in the calibration. Increasing the number of well observed SNe will reduce the statistical errors but, eventually, all systematic errors should be considered.

In our models, the amount of the crucial ${ }^{56} \mathrm{Ni}$ produced is very similar; to some extent this is due to the assumption of a fixed transition density (in the context of delayed detonation explosions). The transition density -or other explosion properties- should be correlated with the initial conditions at the explosion time and hence, with properties of the progenitor. Unfortunately, we are still far from a successfull 3D explosion free of parameters (Gamezo et al. 2005; Röpke \& Hillenbrandt 2005; Bravo \& García-Senz 2006, Plewa et al. 2007).

Acknowledgements This work has been partially supported by the MCyT grant AYA200508013-C03-03, by the Spanish-Italian cooperation INFN-CICYT, by the 2003 PRIN INAF Project and by the Aspen Center for Physics (2007).

\section{References}

[1] D. Branch, W. Romanishing, E. Baron, Astrophys. J., 465, 73 (1996).

[2] C. Badenes, E. Bravo, K.J. Borkowski, I. Domínguez, Astrophys. J., 593, 358 (2003).

[3] E. Bravo, I. Domínguez, J. Isern, R. Canal, P. Höflich, J. Labay, Astronomy and Astrophysics, 269, 187 (1993). 
[4] E. Bravo, E., A. Tornambe, A., I. Domínguez, J. Isern, Astronomy and Astrophysics, 306, 811 (1996).

[5] E. Bravo, D. García-Senz, Astrophys. J., 642, L157 (2006).

[6] A. Chieffi, M. Limongi, O. Straniero Astrophys. J., 502, 737 (1998).

[7] A. Chieffi, I. Domínguez, M. Limongi, O. Straniero, Astrophys. J., 554, 1159 (2001).

[8] I. Domínguez, A. Chieffi, M. Limongi, O. Straniero Astrophys. J., 524, 226 (1999).

[9] I. Domínguez, P. Höflich, O. Straniero, Astrophys. J., 557, 279 (2001).

[10] I. Domínguez, L. Piersanti, E. Bravo, A. Tornambe, O. Straniero, S. Gagliardi, Astrophys. J., 644, 21 (2006).

[11] V.N. Gamezo, A.M. Khokhlov, E.S. Oran, E. S., Astrophys. J., 623, 337 (2005).

[12] M. Hamuy et al., Astron. J., 109, 1 (1995).

[13] M. Hamuy et al., Astron. J., 112, 2391 (1996).

[14] M. Hamuy et al., Astron. J., 120, 1479 (2000).

[15] P. Höflich, Astrophys. J., 443, 89 (1995).

[16] P. Höflich, A. Khokhlov, Astrophys. J., 457, 500 (1996).

[17] P. Höflich, J.C. Wheeler, F.K. Thielemann, Astrophys. J., 495, 617 (1998).

[18] P. Höflich, C. Gerardy, E. Linder et al. Lecture Notes in Physics (review), 635, 203 (2003).

[19] P. Höflich, C. Gerardy, R.A. Fesen, S. Sakai, Astrophys. J., 568, 791 (2002).

[20] V.D. Ivanov, M. Hamuy, P.A. Pinto, Astrophys. J., 542, 588 (2000).

[21] A. Khokhlov, Astrophys. J., 245, 114 (1991).

[22] E.J. Lentz, E. Baron, D. Branch, P.H. Hauschildt, P.E. Nugent, Astrophys. J., 530, 966 (2000).

[23] F. Mannucci et al. Astronomy and Astrophysics, 433, 807 (2005).

[24] F. Mannucci, M. Della Valle, N. Panagia, Monthly Notices of the Royal Astronomical Society, 370, 773 (2006).

[25] S. Perlmutter et al., Astrophys. J., 517, 565, (1999).

[26] L. Piersanti, S. Gagliardi, I. Jr. Iben, A. Tornambe, Astrophys. J., 583, 885 (2003a).

[27] L. Piersanti, S. Gagliardi, I. Jr. Iben, A. Tornambe, Astrophys. J., 598, 1229 (2003b).

[28] M.M. Phillips, Astrophys. J. Lett. 413, L105 (1993).

[29] M.M. Phillips, P. Lira, N.B. Suntzeff, R.A. Schommer, M. Hamuy, J. Maza, Astron. J., 118, 1766 (1999).

[30] T. Plewa, Astrophys. J. Lett., 657, 942 (2007).

[31] A. Riess et al., Astrophys. J., 659, 98 (2007).

[32] F.K. Röpke, W. Hillebrandt, Astronomy and Astrophysics, 431, 635 (2005).

[33] H. Saio, K. Nomoto, Astronomy and Astrophysics, 150, 21S (1985).

[34] H. Saio, K. Nomoto, Astrophys. J., 615, 444 (2004).

[35] B.P. Schmidt et al., Astrophys. J., 507, 460 (1998).

[36] M. Sullivan et al. Astrophys. J., 648, 868 (2006).

[37] J.C. Wheeler, P. Höflich, R.P. Harkness, J. Spyromilio, Astrophys. J., 496, 908 (1998). 